\title{
SENSITIVITY OF TRANSIENT TEMPERATURE FIELD IN THE CASTING DOMAIN WITH RESPECT TO LATENT HEAT PERTURBATIONS
}

\author{
Wioletta Tuzikiewicz \\ Institute of Mathematics, Czestochowa University of Technology \\ Czestochowa, Poland \\ wioletta.tuzikiewicz@im.pcz.pl
}

\begin{abstract}
The mathematical model of thermal processes in the casting domain can be formulated using the fixed domain approach. The energy equation corresponding to this model contains the parameter called a substitute thermal capacity (STC), in which the alloy latent heat appears. The aim of considerations is the application of sensitivity analysis methods for estimation of temperature field changes due to the perturbations of alloy latent heat. In this the analytical form of STC results from the consideration concerning the lever arm model and the Scheil model. The equations determining the sensitivity function are found using the so-called direct approach. At the stage of numerical modeling the finite difference method is used. In the final part of the paper the example of computations is shown.
\end{abstract}

Keywords: alloys solidification, fixed domain approach, substitute thermal capacity, sensitivity analysis, numerical methods

\section{Governing equations}

We consider the following energy equation [1-4]

$$
c(T) \frac{\partial T(x, t)}{\partial t}=\nabla[\lambda(T) \nabla T(x, t)]+L \frac{\partial f_{S}(x, t)}{\partial t}
$$

where $c(T)$ is a volumetric specific heat of casting material, $\lambda(T)$ is a thermal conductivity, $L$ is a volumetric latent heat, $T=T(x, t), f_{S}=f_{S}(x, t)$ denote the temperature and the local volumetric fraction of solid state. One can see that only heat conduction in a casting volume is considered.

Denoting by $T_{L}$ and $T_{S}$ the temperatures corresponding to the beginning and the end of the solidification process and assuming the knowledge of temperaturedependent function $f_{S}$ for $T(x, t) \in\left[T_{S}, T_{L}\right]$ one has

$$
\frac{\partial f_{S}(x, t)}{\partial t}=\frac{\mathrm{d} f_{S}}{\mathrm{~d} T} \frac{\partial T(x, t)}{\partial t}
$$


Introducing this formula to equation (1) one obtains

$$
C(T) \frac{\partial T(x, t)}{\partial t}=\nabla[\lambda(T) \nabla T(x, t)]
$$

where

$$
C(T)=c(T)-L \frac{\mathrm{d} f_{S}(x, t)}{\mathrm{d} t}
$$

is called a substitute thermal capacity. Because for the constant value of $f_{S}$ the derivative $\mathrm{d} f_{S} / \mathrm{d} T=0$, therefore $[3,4]$

$$
C(T)= \begin{cases}c_{L} & T>T_{L} \\ c_{P}-L \frac{\mathrm{d} f_{S}}{\mathrm{~d} T} & T_{S} \leq T \leq T_{L} \\ c_{S} & T<T_{S}\end{cases}
$$

In this place one can also introduce $f_{L}=1-f_{S}$.

In equation (5) $c_{L}, c_{P}, c_{S}$ are the volumetric specific heats of molten metal, mushy zone and solid state sub-domains. So, equation (3) constitutes the model of thermal processes proceeding in the whole, conventionally homogeneous, casting domain.

Considering the typical mathematical description of the foundry process, the energy equation for the casting domain should be supplemented by the similar equation concerning the mould domain, namely

$$
c_{M}(T) \frac{\partial T_{M}(x, t)}{\partial t}=\nabla\left[\lambda_{M}(T) \nabla T_{M}(x, t)\right]
$$

where $c_{M}$ and $\lambda_{M}$ denote the mould volumetric specific heat and mould thermal conductivity.

On the contact surface between the casting and the mould the following boundary condition is given

$$
-\lambda \frac{\partial T(x, t)}{\partial n}=\frac{T(x, t)-T_{m}(x, t)}{R(x, t)}=-\lambda_{M} \frac{\partial T_{M}(x, t)}{\partial n}
$$

where $\partial / \partial n$ is a normal derivative, $R$ is a thermal resistance. For $R=0$ (ideal contact) the last condition takes a form

$$
\left\{\begin{array}{l}
-\lambda \frac{\partial T(x, t)}{\partial n}=-\lambda_{M} \frac{\partial T_{M}(x, t)}{\partial n} \\
T(x, t)=T_{M}(x, t)
\end{array}\right.
$$


On the external surface of the mould the boundary condition in a general form

$$
\Phi\left[T_{M}(x, t), \frac{\partial T_{M}(x, t)}{\partial n}\right]=0
$$

is accepted (in this place the Dirichlet, Neumann or Robin conditions can be taken into account).

The initial temperature distribution is also known

$$
t=0: \quad T(x, 0)=T_{0}(x), T_{M}(x, 0)=T_{M 0}(x)
$$

where $T_{0}$ is the pouring temperature, $T_{M 0}$ is the mould initial temperature.

\section{Mathematical model of the process}

The definitions of substitute thermal capacity presented below result from certain considerations concerning the mass (or volume) balance of alloy component in the casting volume. Assuming that the mass densities of liquid and solid are the same, both approaches give the same results. Let us consider the times $t$ and $t+\Delta t$. Then, the volume balance can be written in the form

$$
\begin{gathered}
V_{S}(t) z_{S}(t)+V_{L}(t) z_{L}(t)= \\
=V_{S}(t+\Delta t) z_{S}(t+\Delta t)+V_{L}(t+\Delta t) z_{L}(t+\Delta t)
\end{gathered}
$$

where $z_{S}, z_{L}$ are the concentrations of alloy component in the solid and liquid phases. The values of $V_{S}, V_{L}$ and $z_{S}, z_{L}$ for time $t+\Delta t$ one can find using the Taylor formula (neglecting the summands of higher order containing $\Delta t^{2}$ and the next ones).

Finally,

$$
V_{S} \frac{\mathrm{d} z_{S}}{\mathrm{~d} t}+\frac{\mathrm{d} V_{S}}{\mathrm{~d} t} z_{S}+V_{L} \frac{\mathrm{d} z_{L}}{\mathrm{~d} t}+\frac{\mathrm{d} V_{L}}{\mathrm{~d} t} z_{L}=0
$$

or, taking into account the definitions of $f_{S}$ and $f_{L}$

$$
f_{S} \frac{\mathrm{d} z_{S}}{\mathrm{~d} t}+\frac{\mathrm{d} f_{S}}{\mathrm{~d} t} z_{S}+f_{L} \frac{\mathrm{d} z_{L}}{\mathrm{~d} t}+\frac{\mathrm{d} f_{L}}{\mathrm{~d} t} z_{L}=0
$$

The partition coefficient $k=z_{S} / z_{L}$ (assuming its constant value) and the dependence $f_{S}=1-f_{L}$ can be introduced and then

$$
\left(1-f_{L}\right) k-\frac{\mathrm{d} f_{L}}{\mathrm{~d} z_{L}} k z_{L}+\frac{\mathrm{d} f_{L}}{\mathrm{~d} z_{L}} z_{L}=0
$$


or

$$
\frac{\mathrm{d} f_{L}}{\mathrm{~d} z_{L}}+\frac{f_{L}}{z_{L}}=-\frac{k}{1-k} \cdot \frac{1}{z_{L}}
$$

The equation (15) is supplemented by the condition $z=z_{0}: f_{L}=1$.

The assumption being that the partition coefficient $k$ is a constant value (the lines $T_{S}$ and $T_{L}$ on the equilibrium diagram are the straight ones and they start from the same point $T_{P}$ ) one obtains the following solution

$$
f_{L}=\frac{z_{0}-k z_{L}}{(1-k) z_{L}}
$$

This formula corresponds to the lever arm model.

Assuming that the derivative $\frac{\mathrm{d} z_{S}}{\mathrm{~d} t}=0$ one has

$$
\frac{\mathrm{d} f_{S}}{\mathrm{~d} t} z_{S}+f_{L} \frac{\mathrm{d} z_{L}}{\mathrm{~d} t}+\frac{\mathrm{d} f_{L}}{\mathrm{~d} t} z_{L}=0
$$

For $z=z_{0}: f_{L}=1$ and then

$$
f_{L}=\left(\frac{z_{0}}{z_{L}}\right)^{\frac{1}{1-k}}
$$

The last equation corresponds to the Scheil model.

The dependences presented below can be introduced to the definition of STC and then one obtains [5]

$$
C(T)=c_{P}-\frac{L\left(T_{0}-T_{P}\right)}{(1-k)\left(T-T_{P}\right)^{2}}
$$

or in the case of the Scheil model

$$
C(T)=c_{P}+\frac{L}{(1-k)\left(T_{P}-T_{0}\right)}\left(\frac{T_{P}-T_{0}}{T_{P}-T}\right)^{\frac{2-k}{1-k}}
$$

One can see that both formulas (19) and (20) contain the latent heat $L$ and the sensitivity analysis with respect to this parameter will be presented in the next section. 


\section{Sensitivity analysis}

The direct approach of the sensitivity model construction relies on the differentiation of differential equations and the boundary-initial conditions with respect to the parameter considered $[5,6]$. The differentiation of energy equation (3) with respect to $L$ gives

$$
\begin{aligned}
& \frac{\partial C(T)}{\partial T} U(x, t) \frac{\partial T(x, t)}{\partial t}+C(T) \frac{\partial U(x, t)}{\partial t}= \\
= & \nabla\left[\frac{\partial \lambda(T)}{\partial T} U(x, t) \nabla T(x, t)+\lambda(T) \nabla U(x, t)\right]
\end{aligned}
$$

where $U(x, t)=\partial T(x, t) / \partial L$.

Assuming that the thermal conductivity is a pieces constant function, one obtains

$$
C(T) \frac{\partial U(x, t)}{\partial t}=\nabla[\lambda(T) \nabla U(x, t)]+Q(x, t)
$$

where

$$
Q(x, t)=-\frac{\partial C(T)}{\partial T} U(x, t) \frac{\partial T(x, t)}{\partial t}
$$

One can see that the last equation is similar to equation (3). The artificial internal heat source contains the coupling factor with (3), the solution of (22) requires the knowledge of the solution to equation (3). In a similar way the other equations creating the sensitivity $U(x, t)$ model can be obtained.

\section{Example of computations}

At the stage of numerical computations the problem of casting solidification made from Al-Si alloy $(2 \% \mathrm{Si})$ has been analyzed. The frame $(2 \mathrm{D}$ problem Figure 1 [7]). The following thermophysical parameters have been introduced [7]: $c_{S}=2.96 \mathrm{MJ} / \mathrm{m}^{3} \mathrm{~K}, c_{L}=3.07, \lambda_{S}=250 \mathrm{~W} / \mathrm{mK}, \lambda_{L}=104, L=990.6 \mathrm{MJ} / \mathrm{m}^{3}, k=0.25$, $T_{P}=660^{\circ} \mathrm{C}$.

The remaining input data and the details concerning the numerical solution of such a problem can be found in paper [7] developed by Szopa, Siedlecki and Wojciechowska.

The example of results obtained corresponds to equation (19). In particular, Figure 2 shows the cooling curves at the points 1, 2, 3 marked in Figure 1. 


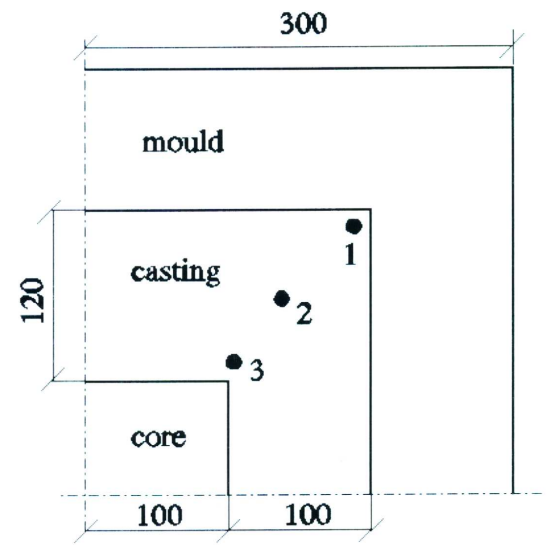

Fig. 1. Frame geometry

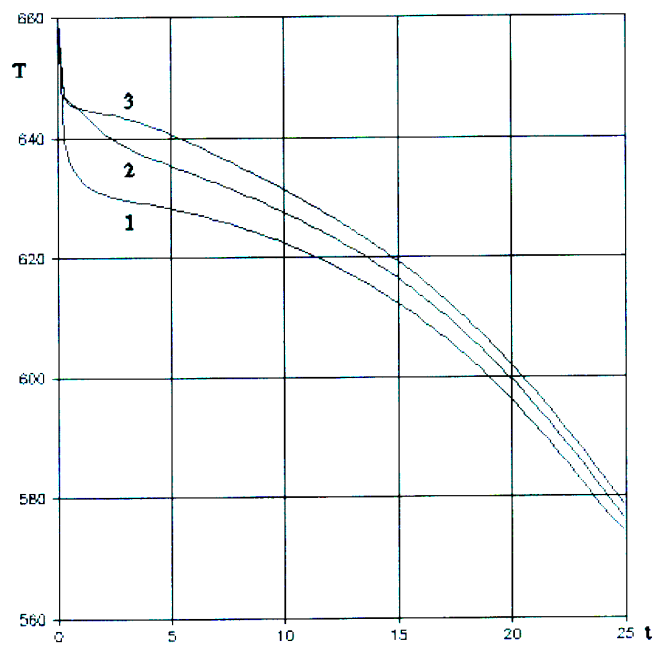

Fig. 2. Cooling curves

The sensitivity analysis with respect to the latent heat has been done for the lever arm model (equation (19)). For the assumed perturbation of $L\left(L_{0} \pm 0.1 L_{0}\right)$, where $L_{0}=990.6 \mathrm{MJ} / \mathrm{m}^{3}$ the maximum disorders of temperature $(5 \mathrm{~K})$ take place at the point 1 (Fig. 1) and they appear in the mushy and solid states sub-domains. For the temperatures $T(x, t)>T_{L}$ the cooling curves are exactly the same, of course.

\section{References}

[1] Mochnacki B., Suchy J.S., Numerical Methods in Computations of Foundry Processes, PFTA, Cracow 1995.

[2] Majchrzak E., Mochnacki B., Suchy J.S., Identification of substitute thermal capacity of solidifying alloy, Journal of Theoretical and Applied Mechanics 2008, 46, 2, 257-268. 
[3] Mochnacki B., Numerical modeling of solidification process (Chapter 24), [in:] Computational Simulations and Applications, Jianping Zhu (ed.), INTECH, 2011, 513-542.

[4] Siedlecki J., Doctoral Thesis, Czestochowa University of Technology, Częstochowa 2002.

[5] Mochnacki B., Definition of alloy substitute thermal capacity using the simple macrosegregation models, Archives of Foundry Engineering 2012, 19, 4, 113-116.

[6] Dems K., Sensitivity analysis in thermal problems, 1: Variation of material parameters within fixed domain, Journal of Thermal Stresses 1996, 9, 303-324.

[7] Szopa R., Siedlecki J., Wojciechowska W., Numerical simulation of solidification process using the Scheil model, Scientific Research of the Institute of Mathematics and Computer Science 2007, $1(6), 253-260$.

[8] Kleiber M., Parameter Sensitivity in Nonlinear Mechanics, J. Wiley \& Sons Ltd., Chichester 1997. 